Service social

Proceedings - 1989 - Actes du symposium, par le Comité de coordination du $11^{e}$ symposium annuel de l'Association for the Advancement of Social Work with Groups, avec la collaboration de Jocelyn Lindsay et Jean-Pierre Landriault, 1392 pages.

\title{
Lise Darveau-Fournier
}

Volume 39, numéro 1, 1990

Le groupe ici et ailleurs

URI : https://id.erudit.org/iderudit/706472ar

DOI : https://doi.org/10.7202/706472ar

Aller au sommaire du numéro

Éditeur(s)

École de service social de l'Université Laval

ISSN

1708-1734 (numérique)

Découvrir la revue

Citer ce compte rendu

Darveau-Fournier, L. (1990). Compte rendu de [Proceedings - 1989 - Actes du symposium, par le Comité de coordination du $11^{e}$ symposium annuel de l'Association for the Advancement of Social Work with Groups, avec la collaboration de Jocelyn Lindsay et Jean-Pierre Landriault, 1392 pages.] Service social, 39(1), 196-200. https://doi.org/10.7202/706472ar d'utilisation que vous pouvez consulter en ligne. 


\section{Note}

* Nous avons eu accès à une version française, datant de 1938 (L'entr'aide, Paris, Alfred Costes éditeur).

Proceedings - 1989 - Actes du symposium (Volumes 1 et 2). Publiés par le Comité de coordination du $11^{\mathrm{e}}$ symposium annuel de I'Association for the Advancement of Social Work with Groups, avec la collaboration de Jocelyn Lindsay et Jean-Pierre Landriault, 1392 pages.

Les Actes regroupent les textes de 81 communications présentées au symposium sur le service social des groupes tenu à Montréal du 26 au 29 octobre 1989.

Les articles - quelques-uns en français mais la majorité en anglais - sont regroupés par sections. Une première division intitulée "Plénières" contient la conférence de Margot Breton ainsi que celle de Beulah Rothman et Catherine Papell. Étant donné l'importance de leur contenu respectif pour l'enseignement et la pratique actuels de l'intervention de groupe, ces conférences ont été traduites pour Service social et reproduites dans le présent numéro sous les titres suivants : "Leçons à tirer de nos traditions en service social des groupes" (p. 13-26); "Service social des groupes en contexte d'autorité " (p. 45-63). Une deuxième section, intitulée " Administration » (p. 47-60) traite de trois aspects à prendre en considération dans la préparation et la réalisation d'un programme de groupe : les difficultés reliées à l'implantation et au développement des groupes, certaines conditions d'efficacité (dont le nombre de membres et d'intervenants, la durée du groupe et les divers frais à prévoir) en rapport avec les coûts du programme, la formation des intervenants et la coanimation. Même si les auteurs, S.S. Korman et D.S. Donofrio, appuient leur réflexion sur leur propre expérience dans un organisme qui offre des groupes de thérapie aux enfants et adolescents émotionnellement perturbés ainsi qu'à leur famille, leurs commentaires demeurent applicables à tout programme de groupe.

Les Actes présentent ensuite huit sections thématiques: personnes âgées; groupes populaires et mouvements sociaux; famille, enfance et adolescence; femmes et hommes; groupes avec besoins spéciaux; santé, santé mentale, personnes handicapées; groupes minoritaires, milieu multiculturel et international; théorie, formation et recherche.

Les différents sujets présentés dans les Actes témoignent de la diversité et de l'étendue de la méthode de groupe. On y discute d'intervention auprès de clientèles très diversifiées, partant des jeunes enfants, des adolescents et de leurs parents, jusqu'aux adultes et aux personnes âgées. De nombreuses problématiques y sont aussi abordées : besoins de répit chez les donneurs de soins aux personnes âgées ou handicapées, problèmes des familles monoparentales, des personnes séparées ou divorcées, des familles en transition, des familles recomposées, des personnes handicapées, des sidéens, des psychiatrisés. On y traite aussi d'isolement social, de violence, de toxicomanie, de délinquance juvénile, d'abus sexuels, etc. L'intervention auprès 
de divers groupes ethniques et de communautés multiculturelles y est aussi discutée.

Je voudrais maintenant $m^{\prime}$ attarder un peu plus sur certains des textes présentés en français. On retrouve d'abord, dans la section " Famille, enfance et adolescence " la présentation d'un service de groupe multifamilial (p. 171$185)$; il s'agit de groupes ouverts offerts à des familles dont les adolescents et adolescentes présentent des difficultés de comportement, de fonctionnement ou d'adaptation. Les auteures, France Allard, Colette Boivin et Monique Cloutier y décrivent sommairement le programme et illustrent particulièrement, avec exemples à l'appui, les étapes de développement d'un de leurs groupes. Elles énoncent aussi quelques conditions, de nature administrative surtout, qu'elles considèrent importantes pour la réussite du programme. Elles rejoignent, dans leur conclusion, un des fondements mêmes du service social des groupes en rappelant que "la plupart des familles possèdent les ressources nécessaires pour apprendre [...] II s'agit de leur en donner l'opportunité " (p. 184). Le texte présenté dans les Actes demeure toutefois très sommaire et laisse le lecteur sur son appétit. En raison de l'intérêt même de cette formule de groupe, je suggère fortement aux personnes qui s'y intéressent de consulter, à titre de complément, le rapport Service de groupe multi-familial, relations parents-adolescents, des mêmes auteures, publié par le Centre de services sociaux du Montréal métropolitain en juin 1988.

Un autre texte porte aussi sur l'intervention de groupe auprès de parents d'adolescents (p. 265-282), cette fois au moyen de groupes fermés. En plus de situer les relations parents-adolescents dans une perspective écologique, les auteurs - Christiane Gagnon et Daniel Turcotte - y discutent de certaines conditions susceptibles de faciliter l'émergence de l'entraide. Leur description du programme expérimenté est claire et présentée de façon systématique; on y retrouve les objectifs et le programme comme tel, de même que les principes qui les sous-tendent, ainsi que le rôle joué par les intervenants. On y rapporte aussi quelques effets du groupe sur les parents. Les auteurs concluent en rappelant la complémentarité de l'aide professionnelle et du réseau d'entraide. Une liste de références intéressantes et pertinentes complète le texte.

Dans la section "Femmes et hommes ", on retrouve un texte de Françoise Alarie qui présente une intervention féministe de groupe pour femmes victimes de violence conjugale (p. 415-437). On y décrit le groupe comme un lieu de support, d'entraide, de solidarité, d'expérimentation de comportements nouveaux mais aussi comme " acte politique [...qui] oblige l'institution à reconnaître la violence comme un problème social, les femmes battues comme un groupe et non plus comme des bénéficiaires souffrant d'une pathologie ou d'une difficulté relationnelle sérieuses. [...II limite aussi] le pouvoir des intervenantes sur les clientes» (p. 419). L'auteure décrit divers groupes offerts aux femmes, de même que les paramètres de l'intervention (clientèle, objectifs, modalités d'intervention). Elle y présente aussi brièvement différents instruments d'intervention et d'évaluation qu'elle utilise, de même que le rôle des coanimatrices. Certains résultats de l'intervention apparaissent en conclusion; malgré un bilan global positif, l'auteure propose un questionnement approprié en rapport avec "l'importance accordée à l'affirmation de soi afin d'abaisser les comportements de victime alors que la responsabilité de la violence est reportée sur les hommes et le contexte sociopolitique ". Elle interroge aussi la pertinence, à court terme, " du travail sur la colère par le biais d'exercices bio-énergétiques" (p. 434). Elle émet enfin certaines hypothèses en rapport avec le haut taux de désistement lors de certains groupes et suggère quelques façons très concrètes de pallier à ces 
difficultés dont, entre autres, l'utilisation d'un instrument de mesure : le Conflict Tactic Scale de Straus'. Une bibliographie contenant plusieurs ouvrages récents complète le texte.

François Legault présente, pour sa part, un modèle d'intervention visant à faciliter le passage d'un groupe d'aide professionnelle à un groupe d'entraide (p. 526-564). L'auteur, se basant à la fois sur différents écrits et sur son expérience auprès de plus de cent cinquante hommes agresseurs, définit $d^{\prime}$ abord la violence conjugale. II discute ensuite des groupes d'entraide puis situe son intervention dans une perspective écologique, en établissant continuellement le lien avec la problématique de l'homme agresseur. II établit aussi des comparaisons intéressantes entre groupe d'aide professionnelle et groupe d'entraide. Le texte de Legault contient de nombreux éléments théoriques qui en rendent parfois la lecture un peu ardue. II a, par contre, l'avantage de mettre le lecteur en contact avec plusieurs publications. La section intitulée "Stratégies et activités " constitue un guide qui peut être très utile au plan de l'intervention directe : on y retrouve un tableau (p. 544-552) qui rend compte de divers comportements et attitudes que peut utiliser le travailleur social à différentes étapes de l'intervention. Ce tableau se fonde sur l'expérimentation déjà faite par Pierrette Bélanger dont Legault s'est aussi inspiré dans sa propre pratique et auquel il a ajouté différents éléments. Une liste de références renfermant à la fois des ouvrages traitant de la problématique concernée et de l'intervention de groupe complète le texte.

Les groupes que nous trouvons le plus souvent dans les différents organismes de santé et de services sociaux s'adressent à ceux et celles que nous considérons comme les "clients", c'est-à-dire qui rencontrent eux-mêmes les problèmes pour lesquels ils consultent. D'autres personnes, souvent intimement reliées aux "clients" et que nous qualifions de "significatives", ont aussi parfois besoin d'aide. C'est le cas des accompagnateurs et accompagnatrices des femmes qui subissent une interruption volontaire de grossesse. Sous le titre " Groupes et avortement : aller au-delà du discours pro-vie et pro-choix... et plus encore » (p. 822-842), Colette Audet et France Soucy décrivent les objectifs et le programme de groupe ouvert qu'elles offrent à ces accompagnateurs et accompagnatrices en vue de les aider à " mieux jouer leur rôle d'aidant naturel " (p. 829). Elles présentent ensuite le groupe " solidarité-post-avortement ", lequel s'adresse aux femmes qui ont subi un avortement volontaire. Les auteures identifient d'abord, à partir de leurs propres observations complétées par une étude de documentation, les principaux stress post-avortement. En plus de décrire les objectifs ainsi que le programme et ses modalités de réalisation, elles appuient leurs choix théoriques et méthodologiques sur de nombreuses références au service social des groupes et à l'approche féministe. Les auteures identifient aussi quelques résultats, dont l'engagement de certaines participantes dans des activités subséquentes à leur expérience de groupe (coanimation d'autres groupes, marrainage).

L'utilisation du petit groupe à différentes fins de changement personnel et social peut rejoindre des clientèles même très handicapées. Louise Noël attire notre attention sur trois expériences de groupe qu'elle a menées auprès de personnes atteintes de déficits physiques importants. Pour chacun des groupes présentés, elle décrit les caractéristiques particulières des membres, les objectifs visés, les moyens utilisés et les principaux résultats obtenus. La description d'activités concrètes utilisées par l'auteure (collage, théâtre, utilisation du miroir, de vidéocassettes, de jeux et de musique, de tableaux de communication comme soutien à la discussion) démontre la possibilité 
d'adapter les moyens d'intervention aux conditions et besoins des membres; on y trouve un encouragement à la créativité des intervenantes et intervenants.

Je voudrais, en terminant, mentionner le texte de Francine Ouellet, présenté dans la section "Théorie, formation et recherche » (p. 1 301-1 329). L'auteure aborde un aspect essentiel de toute intervention : I'évaluation. Malgré que le texte, préparé en fonction d'un atelier, traite de l'évaluation de façon générale et non spécifiquement appliquée au groupe, il peut être utile aux praticiens qui veulent se sensibiliser à cette question. L'auteure présente d'abord diverses considérations en rapport avec l'utilité de l'évaluation, les moments où elle peut s'effectuer (avant, pendant, après l'intervention) ainsi que différentes modalités et techniques qui peuvent être utilisées. Certaines, dont le groupe nominal et l'évaluation sur système unique, y sont décrites. Les exemples proposés sont rattachés à l'intervention de groupe; on retrouve aussi quelques instruments d'évaluation dont une grille d'observation de comportements d'enfants (p. 1313 ), un formulaire d'évaluation (p. 1314-1 315) ainsi qu'une grille d'observation du développement d'un groupe (p. 1316 ) et une échelle d'atteinte d'objectifs (p. 1324). La présentation de tels instruments d'évaluation peut sans doute être utile aux intervenants, en leur rappelant qu'il existe déjà des questionnaires, grilles ou autres dont ils peuvent s'inspirer pour les adapter à leur groupe ou pour en construire de nouveaux.

Les Actes contiennent aussi deux autres textes en français, ceux de Michèle Brousseau et de Hyam Samaha-Kahi. Je ne les commente pas ici étant donné que leur contenu a été repris sous forme d'article dans le présent numéro de Service social. J'ajouterai toutefois que le texte de Mme SamahaKahi, présenté dans les Actes, renferme des descriptions plus détaillées des conditions matérielles de vie des populations déplacées, au Liban.

Le délai généralement important qui existe entre la préparation d'un texte et sa publication constitue une difficulté majeure dans la diffusion des connaissances et expériences. Le Comité de coordination du symposium a voulu éviter ce piège en mettant le plus rapidement possible le contenu des communications à la disposition. des étudiants, praticiens, chercheurs et professeurs. Tous les textes reçus dans les dates prescrites ont été reproduits tels que fournis par leurs auteurs et distribués pendant le symposium. À l'avantage incontestable que constitue cette rapidité d'accès aux documents s'ajoute un contrepoids d'inconvénients : les articles ne sont pas tous d'égale valeur, leur présentation n'est pas uniforme et certains contiennent diverses fautes et erreurs.

Certains déploreront peut-être aussi le fait que près de quatre-vingt-dix pour cent des textes des Actes sont en anglais. Cette situation s'explique toutefois par la présence d'un nombre beaucoup plus grand de travailleurs sociaux américains et canadiens anglais, membres de l'Association for the Advancement of Social Work with Groups, que de francophones et, par conséquent, par une proportion très élevée de communications soumises en anglais.

Malgré ces états de fait, je considère que les Actes constituent une mine de renseignements très utiles à tous ceux et celles qui s'intéressent au service social des groupes. Les articles qu'on y trouve reflètent assez bien, pour la plupart, le thème du symposium : Innovation-tradition, le service social des groupes et le défi du changement sociétal. Les sujets abordés de même que la façon de les traiter sont très diversifiés; les titres des sections de regroupement le démontrent.

Mentionnons enfin que les textes des Actes peuvent rejoindre des lecteurs aux intérêts multiples, non seulement en raison de la variété de sujets 
traités mais aussi à cause des types de contenus qu'on y retrouve : certains articles présentent des notions davantage théoriques, souvent appuyées par des résultats de recherche, d'autres décrivent et discutent d'expériences concrètes d'intervention. De plus, certains se situent davantage dans une perspective thérapeutique alors que d'autres sont nettement orientés vers le soutien social et le développement communautaire.

Lise Darveau-Fournier

École de service social

Université Laval

\begin{abstract}
Note
1 M.A. Straus (1979). "Measuring intrafamily conflict in violence : The conflict tactics (CT) scales », Journal of marriage and family, vol. $41: 75-78$.
\end{abstract}

\title{
Social Work with Groups in Health Settings, par Abraham LURIE, Gary ROSENBERG et Sidney PINSKY (éd.), Prodist, New York, 1982, 124 pages.
}

Ce livre fait suite à un colloque sur l'utilisation des groupes en milieu de santé, qui fut organisé conjointement par les Départements de service social du Long Island Jewish Hillside Medical Center et du Queens Hospital Center de New York.

L'idée de ce symposium est née de la constatation que l'approche de groupe était de plus en plus utilisée en milieu de santé et qu'on lui reconnaissait des valeurs à la fois thérapeutiques et économiques. On reconnaît aussi que les changements majeurs survenus dans le champ de la santé, tels l'augmentation des clientèles ayant des maladies chroniques, l'accent mis sur les droits des bénéficiaires, les coûts très élevés des soins de santé, les enjeux liés à la qualité des soins et à la responsabilisation, et une réduction dans la durée des hospitalisations avec en corollaire un accent sur la planification du congé, sont tous des défis qui obligent à identifier les enjeux et les problèmes qui se posent si on veut développer des services de groupe.

Les participants à la rédaction de ce livre sont connus pour leur contribution théorique au développement du service social des groupes (Alex Gitterman et Beulah Rothman) ou sont des professionnels chevronnés du milieu de la santé (Abraham Lurie, Gary Rosenberg et Sidney Pinsky, éditeurs de l'ouvrage). La douzaine d'articles de ce livre couvre le contenu suivant :

- une partie plus théorique où Alex Gitterman souligne les avantages et les problèmes à développer des approches de groupe en milieu de santé, et touche à la formation, aux buts et à la composition d'un groupe, aux facteurs temps, espace et taille du groupe, de même qu'aux problèmes de recrutement en milieu institutionnel. Beulah Rothman va plus loin en mettant l'accent sur les principes dans l'utilisation du groupe qui favorisent la santé, et sur l'importance de la structure du groupe et du contenu de la vie de groupe. Une application de ces principes est présentée par Elaine Lonergan. 